\title{
Awfulizing: Some Conceptual and Therapeutic Considerations
}

\author{
Windy Dryden ${ }^{1}$
}

Published online: 28 May 2020

(c) Springer Science+Business Media, LLC, part of Springer Nature 2020

\begin{abstract}
In this paper, I will consider the concept of awfulizing, typically seen in REBT theory as a secondary irrational belief that is derived from the primary irrational belief, known as demandingness, or more colloquially as musturbation. I will look at how the concept of awfulizing has been defined in REBT and some of the objections to its use in therapy and beyond. If REBT therapists are going to target awfulizing beliefs for change then this needs to be done carefully and within the context of a good working alliance. I will outline some important considerations that therapists need to be aware of when working with their clients' awfulizing beliefs.
\end{abstract}

Keywords Beliefs $\cdot$ Awfulizing $\cdot$ Non-awfulizing $\cdot$ Sensitivity

\section{Some Important Concepts and Definitions}

For most of his career as the founder and foremost proponent of Rational Emotive Behaviour Therapy, Albert Ellis argued that there were four major irrational beliefs that largely explained the existence of psychological disturbance: musturbation, awfulizing, low frustration tolerance and self/other/life downing ${ }^{1}$ (e.g. Ellis and Joffe Ellis 2011).

\section{Awfulizing is a Secondary Irrational Belief Derived from Musturbation, the Primary Irrational Belief}

Of the four listed irrational beliefs, Ellis, again for most of his career, regarded musturbation as the 'primary' irrational belief and the other three as secondary irrational

\footnotetext{
1 There are alternative terms used for each of these four irrational beliefs to be found in the REBT literature, but for the purposes of this paper I will use the terms listed above in the main body of the paper.
}

Windy Dryden

windydryden@gmail.com

1 Goldsmiths University of London, London, UK 
beliefs derived from the musturbatory core. Thus, in his writings, Ellis would often outline three key musturbatory ideas; "I must do well and have to be approved of by people whom I find important"..."Other people must treat me fairly and nicely"... "The conditions under which I live must be comfortable and free from major hassles" (Ellis and Joffe Ellis 2011: 58). He has not to my knowledge featured three key awfulizing ideas in the same way in any of his writings. For awfulizing beliefs are deemed to stem from musturbatory ideas and the phrase "and it is awful if I don't/ others don't/conditions don't" can be added at the end of each key musturbatory idea outlined above.

\section{How is Awfulizing Defined?}

As a secondary irrational belief, awfulizing has been defined by Ellis as occurring when a person believes that "a bad, unfortunate, or inconvenient circumstance is more than bad, it is the worst it could be $-100 \%$ rotten" (Ellis and Joffe Ellis 2011: 129). At other places, 'awfulsing' means ' $101 \%$ bad' which stems from the idea that something is 'worse than it absolutely must be' (see Dryden and Neenan 2004). So sometimes Ellis puts 'awful' at the extreme of a 0-100\% badness continuum, while at other times he places it in a magical category of its own jumping to $101 \%$ badness and beyond. ${ }^{2}$ However, both $100 \%$ and $101 \%$ + evaluations are both deemed to stem from an implicit primary demand that 'some bad event must not exist' or 'must not be as bad as it is'.

\section{The Four Markers of an awfulizing Belief}

Taking the above into account, there seems to me to be four situations which characterise what an awfulizing belief is trying to get at (Dryden 2021). These are:

- Nothing could be worse.

- The event in question is $100 \%$ bad or worse.

- No good could possibly come from this bad event.

- This event cannot be transcended or surmounted.

\section{The Language of Awfulizing}

In trying to understand the meaning of awfulizing, it is useful to consider what language REBT therapists use with their clients. In the absence of such data, let me consider what language REBT writers tend to use. In addition to the standard, "It's awful that....", we have, "It's terrible that...." It's the end of the world that....." and "It's catastrophic that...." The last is particularly interesting as it equates a catastrophe with something 'awful'. Ellis, in his informal discussions was always clear that catastrophes do exist but awful (particularly the more than $100 \%$ version) does not

$\overline{2}$ Taken from Buzz Lightyear in the 'Toy Story' films whose slogan was 'to infinity and beyond'. 
and cannot exist, My own view is that in REBT awfulizing is a belief (at ' $\mathrm{B}$ ') and catastrophizing refers to highly distorted cognitive consequences (at ' $C$ ') of this and other irrational beliefs (Dryden 2013). ${ }^{3}$

\section{The Importance of the 'Critical A' or Adversity in Discussing Awfulizing}

Modern REBT has moved away from regarding the 'A' in the 'ABC' framework as an 'activating event' and towards the idea that it is best regarded as an 'adversity'. The latter term points to the subjective nature of the ' $A$ ' sometimes referred to as the 'critical A'. The 'critical A' is the aspect of the situation that the person is in that they are most disturbed about. This is what constitutes the adversity for the person. If, and when, a therapist comes to discuss the role and status of an awfulizing belief with a client, it is paramount in my view for that therapist to discover what precisely the client is disturbed about in the situation in question.

Let's take the present pandemic gripping the world at present, the coronavirus. ${ }^{4}$ What precisely may a person be disturbed about here? The following are 10 possibilities $^{5}$ :

- Death.

- Painful death.

- Being isolated in one's dwelling.

- Having one's freedom curtailed.

- Not being able to see/show affection to loved ones.

- Not knowing if one is going to contract the virus.

- Not knowing what is going to happen to one's job.

- Being ill.

- Being very close to relatives that one strongly dislikes.

- Running out of food and other provisions.

If we are trying to help the public deal with their anxiety in the context of the coronavirus pandemic, we will struggle to help them if we don't know precisely what they are disturbed about. In short, what does the person think is 'awful'. We need to discover this to help them take the horror out of their appraisals.

\section{What is a Non-awfulizing Belief?}

Having discussed the nature and features of an awfulizing belief, let me do the same with a non-awfulizing belief. If an awfulizing is a secondary irrational belief that is derived from a primary irrational belief known as musturbation, then a

\footnotetext{
${ }^{3}$ See an interesting paper by Waltman and Palermo (2019) on the differences between awfulizing and catastrophizing.

4 Recently a large supermarket completely sold out of all brands of beer save one-Corona beer!.

5 There are, of course, more.
} 
non-awfulizing belief is a secondary rational belief that is derived from a primary rational belief that I will call here a non-dogmatic preference.

\section{The Four Markers of a Non-awfulizing Belief}

Taking the above into account, there seems to me to be four situations which characterise what a non-awfulizing belief is trying to get at (Dryden 2021). These are:

- Things could always be worse.

- The event in question is less than $100 \%$ bad.

- Good can come from this bad event and.

- This event can be transcended or surmounted.

\section{The Language of Non-awfulizing}

The language of a non-awfulizing belief shows that when a person holds such a belief that they assert the badness of the adversity, but negate the 'awfulness' of it (e.g. "It's bad, but not awful/terrible/the end of the world). The more aversive the adversity, the stronger the evaluation of badness is.

\section{Therapeutic Considerations}

Helping clients to identify and deal effectively with their awfulizing beliefs requires a fair measure of therapeutic skill, particularly when the adversities that they face are real and highly aversive. In addressing this issue, I will make use of my expanded use of Bordin's (1979) tripartite model of the working alliance (Dryden 2011).

\section{The Importance of the Working Alliance and Questioning Awfulizing Beliefs}

Bordin (1979) argued that there are three components of the working alliance:

- Bonds (the interpersonal connectedness between therapist and client).

- Goals (the objective of therapy).

- Tasks (what the therapist and client need to do so that the client achieves their goals).

Latterly I added a fourth component that I called:

- Views (the understandings that therapist and client have about salient aspects of therapy)—Dryden (2011).

Using my expanded model of the working alliance, when it comes to dealing with awfulizing beliefs, the therapist and client need to already have established a trusting and well-bonded relationship. They need to have shared views of (a) what an 
awfulizing belief is and the role it plays in the client's problem and (b) what a nonawfulizing belief and the role it plays in helping the client to achieve their goal. The skills with which the therapist addresses these issues and how they equip the client to implement a non-awfulizing belief outside the session concern the tasks of therapy in this respect.

What follows from the above is that problems that therapists experience in helping clients to stand back and question their awfulizing beliefs occur in part:

- When the therapist and client do not share an accurate view on the nature of an awfulizing belief and/or the client does not understand what constitutes a nonawfulizing belief.

- The client does not understand how their awfulizing belief is implicated in their problem and/or how their alternative non-awfulizing belief can help them to achieve goal.

- The client perceives the therapist as insensitive in questioning their awfulizing belief. The risk of this is increased the more aversive the client's adversity is at 'A'.

\section{Sensitivity in Questioning Awfulizing Beliefs}

While it is the case that REBT therapists should advisedly show sensitivity in carrying out a range of therapeutic tasks, such sensitivity should particularly be to the fore when helping clients to stand back and question their awfulizing beliefs. This is especially the case when their adversities are highly aversive such as the sudden loss of a loved one to the coronavirus, being told one has a terminal illness or losing one's home and all one's possessions in a tsunami.

Here are the ingredients of such sensitivity.

\section{Validating the Aversive Nature of the Adversity}

If one considers the full form of both an awfulizing belief and a non-awfulizing belief, it is apparent that these two beliefs have a common root which is what I call the 'evaluation of badness' component of the belief. Thus:

- Awfulizing belief 'It is tragic that I lost all my possessions in a tsunami and therefore it is the end of the world'.

- Non-awfulizing belief 'It is tragic that I lost all of my possessions in a tsunami, but however bad it is, it is not the end of the world for me'.

Validating the aversive nature of the adversity means empathising with the client concerning the 'evaluation of badness' component of their belief which in this case is the tragic nature of the adversity that they have experienced. This can be done whether they are holding an awfulizing belief or a non-awfulizing belief. In validating the evaluation of badness component of an awfulizing belief, the therapist needs to take care not to validate the awfulizing component. 
One effective way of doing this is to help the client see that it is tragic that they have lost everything in a tsunami and that they have understandably added the ingredient of 'horror' to the tragedy. I first help them to see what the consequences are of 'tragedy with horror' and 'tragedy without horror'. Once I have done this then I help them to see that they have the option to move forward with the belief 'tragedy with horror' or the belief 'tragedy without horror'. If they choose the latter, then I help them to do this. If they choose the former, I will strive to understand what their doubts, reservations or objections (DROs) are which lead them to want to hold on to the horror component or to be reluctant to move forward without the horror component (see later).

\section{Helping Clients to Embrace Strong Healthy Negative Emotions}

In my view, one of the strengths of the REBT model is its concept of healthy negative emotions (HNEs). This states that it is constructive for someone to experience a very strong negative emotion in response to a highly aversive adversity and that it is possible for the person to do this and for the emotion to be healthy. Elsewhere, I have argued that the health of a negative emotion is determined by the behaviours (including action tendencies) and thinking that accompanies the emotion (Dryden 2012). Helping the client discussed above to see that they can still feel painful, but healthy negative emotions in response to their great loss can be liberating and that they can achieve this by taking the horror out of the tragedy, not the tragedy out of the tragedy. Validating the experience of intense HNEs while communicating an understanding why the person may experience intense UNEs without validating them is particularly tricky but can yield powerfully constructive results.

\section{Understanding and Using the Concepts of 'Time' and 'Process' in Dealing with Awfulizing Beliefs}

Unless one has a black belt in rational thinking, it is understandable that a person would respond to a highly aversive adversity with an awfulizing belief. However, just because one does so initially does not mean that the person will necessarily continue to do so over time. What follows from this is that this time-limited irrationality (Dryden and Neenan 2015), may not be targeted for change unless the person has been stuck in viewing things from an awfulizing perspective and can't get out of this stuckness.

In the latter case, the client should be helped to see that they do have an alternative to hand (i.e. a non-awfulizing belief). If the therapist can involve the client in a discussion about what holding a non-awfulizing belief involves then the client is offered a plausible alternative. If they choose to develop the alternative non-awfulizing belief then they can be helped to see that initially they still might go to their awfulizing belief first but they can accept that this may be their first response and they can choose to respond to this first response with arguments designed to weaken their conviction in their awfulizing belief and to strengthen their conviction in their non-awfulizing belief. In short, the client can be helped to see that there is a process 
between holding an awfulizing belief and developing a non-awfulizing belief and this may mean that the client may begin with an awfulizing belief, but to view this as the initial part of a series of responses and that if they engage with the questioning process (known traditionally as 'disputing' in REBT) then they may end up with holding a non-awfulizing belief.

\section{The Use of Time Projection}

One very effective imagery method that can be used in the questioning of an awfulizing belief is that of time projection (Lazarus 1984). When a client holds an awfulizing belief about an adversity, then they tend to stop thinking about it and therefore cannot see beyond its 'awfulness'. The purpose of time projection is to enable clients to see vividly that time and the world continue after the 'awful' event' has occurred. Thus, for example, I once saw a Malaysian student for counselling whose tuition fees were paid for by his village and who held an awfulizing belief about facing his fellow villagers were he to fail his exams. I helped him to imagine his return to his village while experiencing shame and as time was gradually advanced forward via imagery, he began to see that it was likely that his fellow villagers would eventually come to adopt a compassionate viewpoint toward him, and even if they did not, he could always live happily in another part of the country, or in another part of the world. His connection with 'time' helped him to unfreeze this event and then process it which in turn enabled him to consider his worst case scenario and see that he could eventually take the horror out of letting his village down, but not the badness out of it, as discussed above.

Once the client has through time projection been helped to see that what was once regarded as awful at one time is not awful at a later time, this opens the door to a discussion with the client of perhaps learning to take the horror out of an adversity earlier in the process of unfolding time.

\section{Focus on What is Persuasive to the Client}

DiGiuseppe (1991) showed almost three decades ago that when REB therapists questioned or disputed their clients' irrational beliefs then they used three types of arguments in doing so: empirical, logical and pragmatic. One of the dangers of using this formulation is that therapists carry out such questioning in an overly structured and prescribed way which will eventually have a negative effect on clients on the receiving end of such 'formulaic disputing'. Instead, I recommend that the emphasis is placed on helping the client to develop arguments that they find persuasive and which target awfulizing beliefs. Here are four ways of doing this:

\section{Use Previously Persuasive Arguments}

Encourage the client to use arguments that they have previously found persuasive in the therapeutic process and gauge the effect of doing this on the client's awfulizing belief. 


\section{Develop Persuasive Arguments}

Ask the client directly what arguments they would find persuasive in what might be called the de-awfulizing process. This may seem naïve, but George Kelly used to advise psychologists, 'If you want to know anything about the client, ask him. He might just tell you'.

\section{Use the Client's Own Prior Experience of De-awfulizing}

It may be the case that the client has had prior experience of evaluating something as 'awful' in their lives and then having a later opportunity to re-evaluate this so that they ended up with a healthier appraisal. It is useful to find out about such experiences and 'mine' them for the gold of what the person found persuasive in their own reappraisal experience. When you have done this, you can encourage the client to bring what they learned from the previous experience and apply this to the current situation.

\section{Teach Your Children Well}

Sometimes it is useful to put the client in the role of questioner or disputer and a common way of doing this is to ask the client to imagine that they have the opportunity to advise a group of children whether to hold an awfulizing belief or a nonawfulizing belief about some major adversity. Assuming that the client chooses to teach the children to use the non-awfulizing belief, the role of the therapist is to list the arguments the client develops in this scenario and later to invite them to use these arguments with their own awfulizing belief. Particular attention, however, should be placed on the possibility that the client regards themself as an exception, meaning that the arguments that they have used with the group of children either do not apply to or will not work with themself. This often marks the beginning of a useful discussion which reveals important subtleties of the client's belief system.

\section{Arriving at Non-awfulizing Via Disputing Demanding Beliefs and Low Frustration Tolerance Beliefs}

So far in this paper, I have discussed direct ways of helping clients to question their awfulizing beliefs. It is also possible to help clients to see that something that they have evaluated as being 'awful' is, in fact, bad but not awful, but to do so indirectly.

\section{Disputing Musturbatory Beliefs}

As I discussed earlier in this paper, REBT theory argues that awfulizing beliefs stem from musturbatory beliefs and that non-awfulizing beliefs stem from non-dogmatic preferences. If this part of theory is valid then, it should be possible to help a person develop a non-awfulizing belief by first helping them question their musturbatory belief and develop conviction in their alternative non-dogmatic preference. This 
would be particularly the case if the person's conviction in their new non-dogmatic preference was strong.

\section{Disputing Low Frustration Tolerance (LFT) Beliefs}

Another indirect way of helping a client develop a non-awfulizing belief is to first help them to dispute their associated low frustration tolerance (LFT) belief and develop a high frustration tolerance (HFT) belief. Seasoned REB therapists observe that it is easier to help someone take the horror away from an adversity, once they see clearly that they can tolerate the adversity and it is worth it to them to do so.

\section{Identifying and Dealing with Doubts, Reservations and Objections to Awfulizing Beliefs and Non-awfulizing Beliefs: Clients and Therapists}

REBT has a number of concepts that in the course of therapy therapists need to make clear to their clients. Working effectively with these concepts requires therapists to have confidence in relevant concepts to explain sufficiently their clients' disturbance and that if these particular concepts are targeted for change and changed then the clients will respond healthily to life's adversities. If therapists have doubts about the role of awfulizing beliefs in the REBT process and if, in particular, they shy away from dealing with them in therapy, then they need an opportunity to reflect on what doubts, reservations and objections (DROs) they have to this concept. We don't have any information about what DROs REB therapists have about salient aspects of the REB therapeutic process, including awfulizing beliefs and this could be a fruitful line for fruitful inquiry. However, clinically, we encounter clients who do have DROs to adopting a non-awfulizing belief and to giving up an awfulizing belief. The following two DROs are taken from Dryden (2001).

Doubt 1: My awfulizing belief shows that what has happened to me is tragic while the non-awfulizing belief makes light of this tragedy. Therefore, if I surrender my awfulizing belief in favour of the non-awfulizing alternative, I am making light of what is tragic about my life.

Response: This is a commonly expressed doubt, but one that is based on a misconception of what constitutes both an awfulizing belief and a non-awfulizing belief. In order to make my point, I am going to make an important distinction between the terms 'tragic' and 'awful' as they are used in REBT. 'Tragic' refers to something that has happened in your life that is highly aversive and which has changed your life for the worse, but has not irrevocably ruined it. Although the event is "tragic", you can transcend it and go on to live a life with some meaning and happiness. "Awful", on the other hand, means that something has happened to you that has irrevocably ruined your life which you cannot transcend. As a result, your life is devoid of meaning and the possibility of happiness.

Holding a non-awfulizing belief enables you to acknowledge that you have experienced something that is tragic, but not awful according to the above definition. 
This belief helps you to come to terms with the tragedy and to get on rebuilding your life, whereas holding an awfulizing belief means that you think that effectively your life is over and there is nothing that you can to do rebuild it.

I hope that you can see from this discussion that your non-awfulizing does not make light of any tragedy that you befall you. Indeed, it helps you to acknowledge that a tragedy has happened to you, but gives you hope that you can rebuild your life. While your awfulizing belief shows that what has happened to you is tragic, it turns a tragedy into an end of the world experience which you can never get over. Therefore, if you surrender your awfulizing belief in favour of the non-awfulizing alternative, you are not making light of what is tragic about my life. You are acknowledging the tragedy and helping yourself to transcend it and to move on with your life.

Doubt 2: My awfulizing belief sensibly protects me from threat, while my nonawfulizing needlessly exposes me to it.

Response: This doubt refers to situations where you experience anxiety. According to REBT theory if you hold an awfulizing belief about something that you perceive to be a threat then you will experience anxiety. You will then tend to act to protect yourself from this threat usually by avoiding the threat. This unfortunately means that you will not be able to discover whether or not your perception of threat is accurate. Also, by avoiding the "threat" you will not develop constructive ways of dealing with it should it turn out to be an accurate threat.

By contrast, if you hold a non-awfulizing belief about something that you perceive to be a threat then you will experience concern. You will then tend to act to protect yourself only if the threat turns out to be real and you are unable to take constructive action to deal effectively with it. This means that you will not avoid the threat the moment that you perceive the situation to be threatening. Rather, you will stay in the situation to test out your hunch that you are facing a threat.

Thus, your awfulizing belief may protect you from threat, but not in a sensible way. In fact, because it influences you to take avoidant action as soon as you perceive a threat, you are likely to continue to perceive threat where none may exist. Also, because you are don't learn to take effective action to deal with threat, you are likely to continue to perceive threat in similar situations. In short, your awfulizing belief may protect you from threat in the short term, it increases the likelihood that you will perceive threat in the longer term.

Also, your non-awfulizing belief does not needlessly expose you to threat. It helps you to remain in the situation long enough to determine whether protective or other action is necessary and if it is it helps to determine what action to take.

In facing a serious pandemic like the present coronavirus situation, is there anything to be gained by adding 'horror' to crisis? The answer is clearly no. REB therapists need to find clear and persuasive ways of disseminating this idea to communities across the world to help preserve the mental health of nations. It is my hope that this paper may go some way in clarifying some of the issues concerning awfulizing to enable us to do just this. 


\section{Compliance with Ethical Standards}

Conflict of interest There is no conflict of interest.

\section{References}

Bordin, E. S. (1979). The generalizability of the psychoanalytic concept of the working alliance. Psychotherapy: Theory, Research and Practice, 16, 252-260.

DiGiuseppe, R. (1991). Comprehensive cognitive disputing in rational-emotive therapy. In M. Bernard (Ed.), Using rational-emotive therapy effectively (pp. 151-195). New York: Plenum.

Dryden, W. (2001). Reason to change: A rational emotive behaviour therapy (REBT) workbook. Hove: Brunner-Routledge.

Dryden, W. (2011). Counselling in a nutshell (2nd ed.). London: Sage.

Dryden, W. (2012). Dealing with emotional problems using rational-Emotive cognitive behaviour therapy: A practitioner's guide. Hove, East Sussex: Routledge.

Dryden, W. (2013). The ABCs of REBT: Perspectives on conceptualization. New York: Springer.

Dryden, W. (2021). Dealing with emotional problems in coaching: A rational-emotive and cognitive behaviour therapy (RECBT) approach (2nd ed.). Abingdon, Oxon: Routledge.

Dryden, W., \& Neenan, M. (2004). Counselling individuals: A rational emotive behavioural handbook (4th ed.). London: Whurr.

Dryden, W., \& Neenan, M. (2015). Rational emotive behaviour therapy: 100 key points and techniques (2nd ed.). Hove, East Sussex: Routledge.

Ellis, A., \& Joffe Ellis, D. (2011). Rational emotive behavior therapy. Washington, DC: American Psychological Association.

Lazarus, A. A. (1984). In the mind's eye. New York: Guilford.

Waltman, S. H., \& Palermo, A. (2019). Theoretical overlap and distinction between rational emotive behavior therapy's awfulizing and cognitive therapy's catastrophizing. Mental Health Review Journal, 24(1), 44-50.

Publisher's Note Springer Nature remains neutral with regard to jurisdictional claims in published maps and institutional affiliations. 